\title{
Contraction of an air disk caught between two different liquids
}

\author{
M.-J. Thoraval and S. T. Thoroddsen \\ Division of Physical Sciences and Engineering and Clean Combustion Research Center, King Abdullah University of Science and Technology, \\ Thuwal 23955-6900, Saudi Arabia
}

(Received 4 September 2013; published 17 December 2013)

\begin{abstract}
When a drop impacts a pool of liquid it entraps a thin disk of air under its center. This disk contracts rapidly into a bubble to minimize surface energy. Herein we use ultra-high-speed imaging to measure the contraction speed of this disk when the drop and pool are of different liquids. For miscible liquids the contraction rate is governed by the weaker of the two surface tensions. Some undulations are observed on the edge of the disk for a water drop impacting a pool of water, but not on a pool of lower surface tension. Similar results are observed for a pair of immiscible liquids.
\end{abstract}

DOI: 10.1103/PhysRevE.88.061001

PACS number(s): 47.55.D-

Introduction. The impact of a drop onto a pool surface always entraps a thin disk of air under the center of the drop [1-3]. This air disk is produced by the lubrication pressure in the air, which flattens the drop and causes contact along the rim of this air disk [3-8]. For high-impact velocity the size and thickness of the air disk is determined by drop shape, pool depth, and primarily the impact Reynolds number using inertia of the liquid, but viscosity of the gas $[3,5,6,9]$. For a typical large raindrop, this disk is exceedingly thin, $\sim 1 \mu \mathrm{m}$ thick and $\sim 400 \mu \mathrm{m}$ in diameter. The subsequent contraction of this disk to minimize surface energy lasts $\sim 100 \mu$ s and can be modeled based on capillary-inertial dynamics [3]. Oguz and Prosperetti [10] showed that by balancing dynamic and capillary pressures, the edge of a thin layer of air contracts at a velocity $u \sim \sqrt{\sigma / \rho \delta}$ with a proportionality constant close to 1. Here $\sigma$ is the surface tension, $\rho$ the liquid density, and $\delta$ the air sheet thickness. This is essentially the same as for liquid sheets in air [11-17]. By using this result and assuming that the disk remains of uniform but growing thickness $\delta(t)$ during the contraction, it is found that the radius of the air disk $R(t)$ reduces exponentially in time $[3,4]$

$$
R(t)=R_{0} \exp \left(-C \sqrt{\pi \sigma / \rho \mathcal{V}_{\text {tot }}} t\right)
$$

where $R_{0}$ is the initial radius of the air disk and $\mathcal{V}_{\text {tot }}$ is the total volume of air entrapped.

Similar disk entrapment and contraction dynamics are also observed for drops impacting a solid surface [4,18-26]. Recent work by Liu et al. [27] has modified the contraction rate model by including a thicker rim.

Herein we study a slightly different configuration, where the drop and the pool consist of different liquids. The aim is to determine how the two surface tensions control the contraction dynamics. When the water drop contacts the lower- $\sigma$ ethanol pool along a circular rim, it suddenly produces an unbalanced stress at the free surface, where the two liquids meet. Strong Marangoni forces thereby created by the spatial variations in surface tension should pull a thin layer of the lower surface tension liquid over the surface of the entrapped air disk [28-30].

Methods. Figure 1 shows the experimental setup. We view the impact from below through a bottom glass plate. We use in all cases a water drop, with equivalent diameter $D=\left(D_{v} D_{h}^{2}\right)^{1 / 3}=4.67 \mathrm{~mm}$, where $D_{v}$ and $D_{h}$ are the vertical and horizontal diameters at impact. The drop is released from a nozzle at a height above the pool between $H=42$ and $45 \mathrm{~cm}$. The drop oscillations give an oblate to spherical drop at impact [8]. The pool is water, ethanol, or perfluorohexane liquid, with properties listed in Table I. The fluorinated liquid has vastly different properties than water and as such can probe the effects of the much lower surface tension in the immiscible case.

The impact is characterized by Reynolds and Weber numbers

$$
\mathrm{Re}=\frac{\rho D U}{\mu} \simeq 12900, \quad \mathrm{We}=\frac{\rho D U^{2}}{\sigma} \simeq 500,
$$

where $U \simeq 2.8 \mathrm{~m} / \mathrm{s}$ is the drop impact velocity and $\rho, \mu$, and $\sigma$ are, respectively, the density, dynamic viscosity, and surface tension of the water drop.

High-speed imaging was done with Photron SA-5 at frame rates up to 100 kiloframes/s $(\mathrm{kfps})$ and $1 \times 10^{6} \mathrm{fps}$ was accomplished with the Shimadzu Hypervision [31]. In combination with a long-distance microscope (Leica APO 16), we reach a pixel resolution of $4 \mu \mathrm{m}$. Cool lighting (Sumita) focused by the drop itself gave sufficient illumination intensity for the highest frame rates.

The shallow pool is confined to a small circular dish, formed by a circular strip of plastic glued onto a 2-mm-thick glass plate. The depth of the pool layer is calculated by assuming a uniform layer thickness over the $10-\mathrm{cm}$ dish. A fixed amount of fresh liquid is deposited from a syringe, just before each impact. The layer thickness $h$ is kept between $250 \mu \mathrm{m}$ and

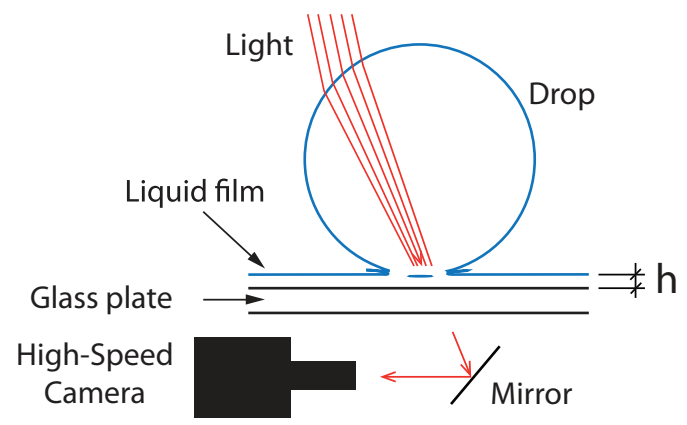

FIG. 1. (Color online) Schematic of the experimental setup, where a water drop impacts a liquid film. The central air disk is imaged through a bottom glass plate. 
TABLE I. Properties of the different liquids.

\begin{tabular}{lccc}
\hline \hline Liquid & $\begin{array}{c}\text { Density } \\
\rho\left(\mathrm{g} / \mathrm{cm}^{3}\right)\end{array}$ & $\begin{array}{c}\text { Viscosity } \\
\mu(\mathrm{cP})\end{array}$ & $\begin{array}{c}\text { Surface tension } \\
\sigma(\mathrm{dyn} / \mathrm{cm})\end{array}$ \\
\hline Distilled water & 0.996 & 1.004 & 72.5 \\
Ethanol & 0.789 & 1.19 & 23.2 \\
Perfluorohexane & 1.710 & 1.1 & 11.9 \\
\hline \hline
\end{tabular}

$1 \mathrm{~mm}$. We believe this depth does not affect the contraction rate of the disk, as the interface distance from the wall is much larger than the air layer thickness and the typical viscous length scale $\ell_{v} \simeq \sqrt{v T} \sim 10 \mu \mathrm{m}$. Experiments with half this film thickness show minimal effects on the dynamics [32].

Contraction speed. Figure 2 shows frames from typical video sequences of the air disk contraction. See also Ref. [34]. Figure 3 shows the corresponding radius vs time for the air disk between miscible liquids. It compares air disks entrapped under identical water drops impacting a water pool versus an ethanol pool. The model in Eq. (1) shows clearly that the contraction of the disk between water and ethanol is governed by the much weaker surface tension of the ethanol rather than that of the water. Here we use the same empirical factor $C=$ 0.94 as determined for air-disk contraction in identical liquids in [3], with no other adjustable parameters.

Immiscible liquids. Figures 2(d) and 4 show the contraction of an air disk between immiscible liquids, i.e., when the water drop impacts a perfluorohexane film. Again we observe the contraction to be controlled by the perfluorohexane's properties, which has the much lower $\sigma$. Using the water properties (solid curve), one predicts much faster contraction than observed. However, here there is an additional complication, as there are three interfacial tensions: two between the individual liquids and air $\left(\sigma_{w a}\right.$ and $\left.\sigma_{p a}\right)$ and in addition the interfacial tension between the two liquids $\left(\sigma_{w p}=48 \mathrm{dyn} / \mathrm{cm}\right)$ and the presence of a triple line, where their interface meets the air.

The inset in Fig. 4 provides a possible configuration where the triple line has been pulled along the air-water interface,

(a)

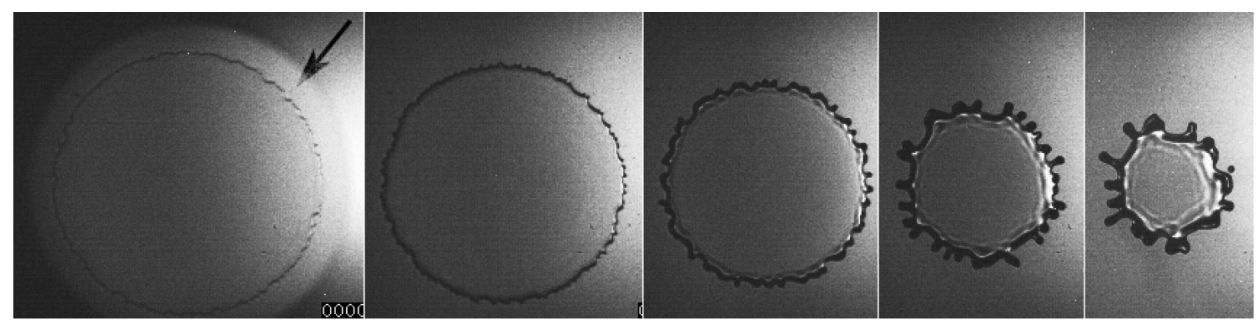

(b)

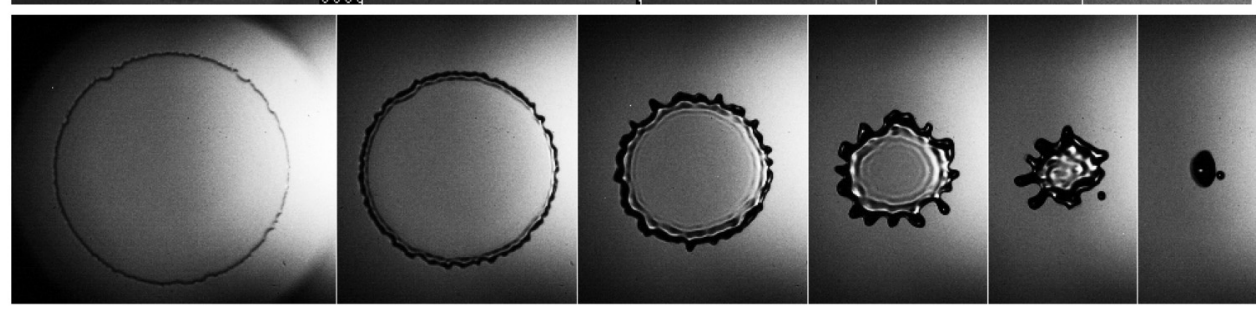

(c)

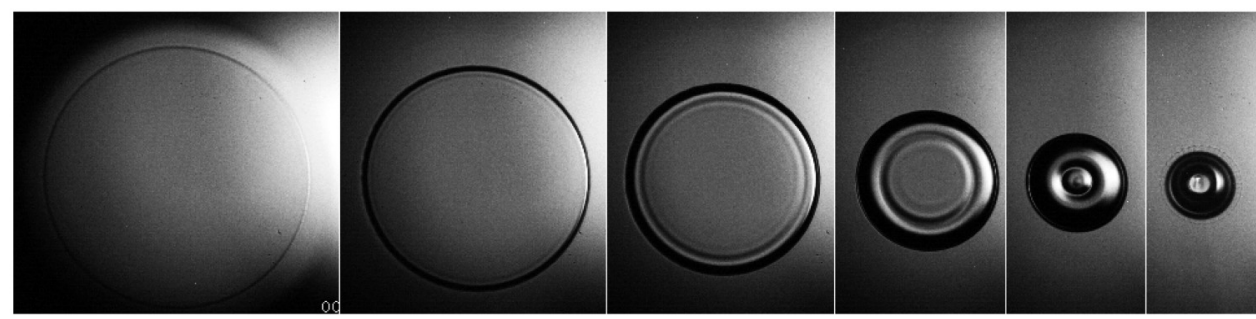

(d)
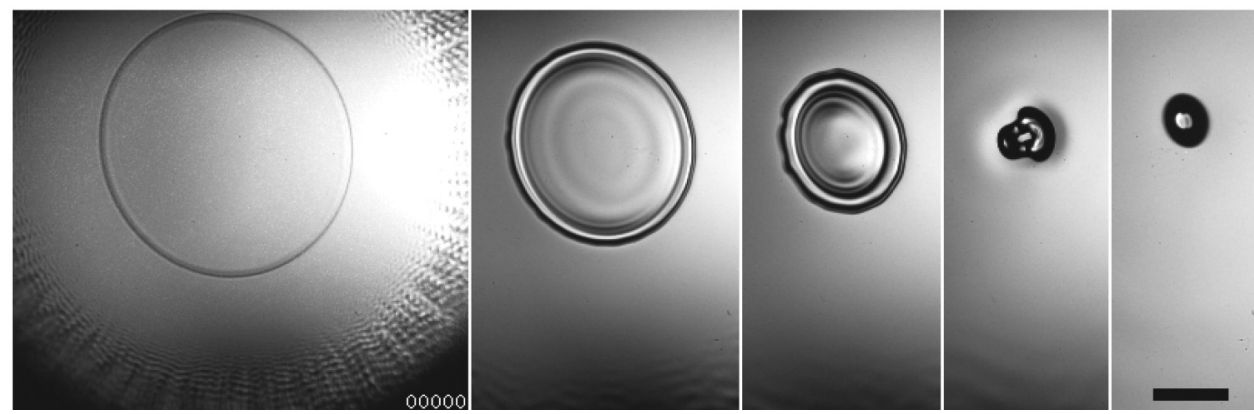

FIG. 2. Typical contraction of the air disk for a water drop (a) and (b) impacting a water layer $(h=1.0 \mathrm{~mm})$. (a) Frames from $1 \times 10^{6} \mathrm{fps}$ video, shown at 1, 7, 18, 36, and $51 \mu$ s after the first contact. (b) Frames at 3, 15, 33, 53, 73, and $141 \mu$ s. (c) Impact onto an ethanol layer $(h=1.0 \mathrm{~mm})$. Frames are shown at $1,21,45,87,125$, and $163 \mu \mathrm{s}$. (d) Water drop impacting a layer of Perfluorohexane, $h=250 \mu \mathrm{m}$. Frames are shown at $\simeq 12,92,172,308$, and $748 \mu \mathrm{s}$. Instabilities are visible on the outer edge of the liquid contact $[8,32,33]$. The scale bar is $200 \mu \mathrm{m}$ long. See Ref. [34]. 


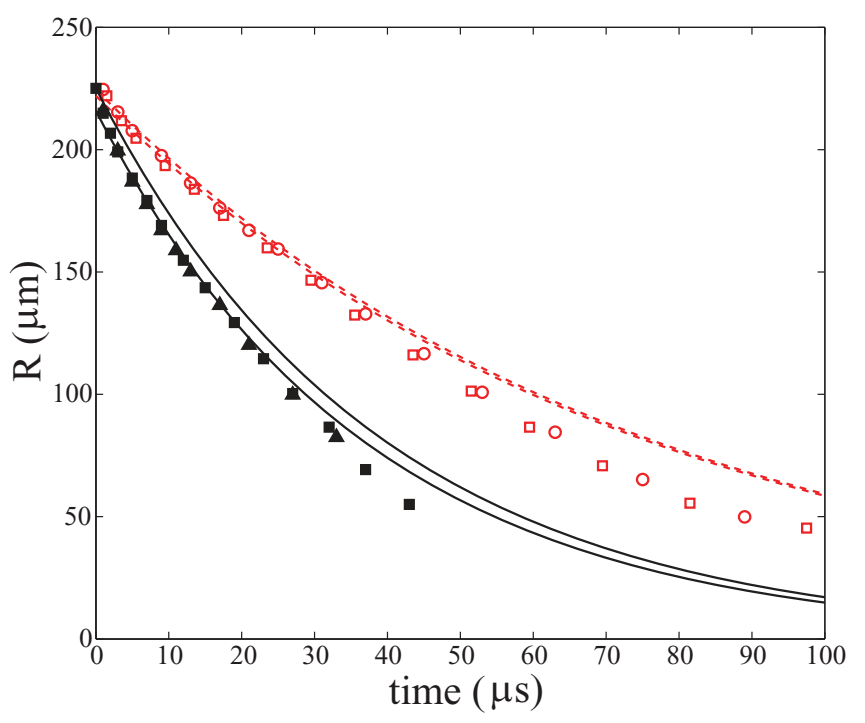

FIG. 3. (Color online) Contraction of the central air disk as a function of time (with $h=250 \mu \mathrm{m}$ and $H=45 \mathrm{~cm}$, giving $\mathrm{Re}=$ 12900 and $\mathrm{We}=500$ for water drop properties, and $D_{v} / D_{h}=0.98$ ). Water drops impacting a water film (closed symbols) and ethanol film (open symbols). The solid lines are from Eq. (1) using water properties and the dashed line is from using ethanol properties, with $C=0.94$. The different symbols correspond to two realizations for each case. The corresponding two lines are calculated with the slightly different initial diameter and thickness of the air disks.

thereby exposing the edge of the disk to the perfluorohexane surface tension to drive the overall contraction. In this scenario the net surface tension pulling at the triple line $\Delta \sigma \geqslant \sigma_{w a}-$ $\sigma_{w p}-\sigma_{p a} \geqslant 12.6 \mathrm{dyn} / \mathrm{cm}$, which is clearly larger than the perfluorohexane surface tension $\sigma_{p a}=11.9 \mathrm{dyn} / \mathrm{cm}$. Clearly,

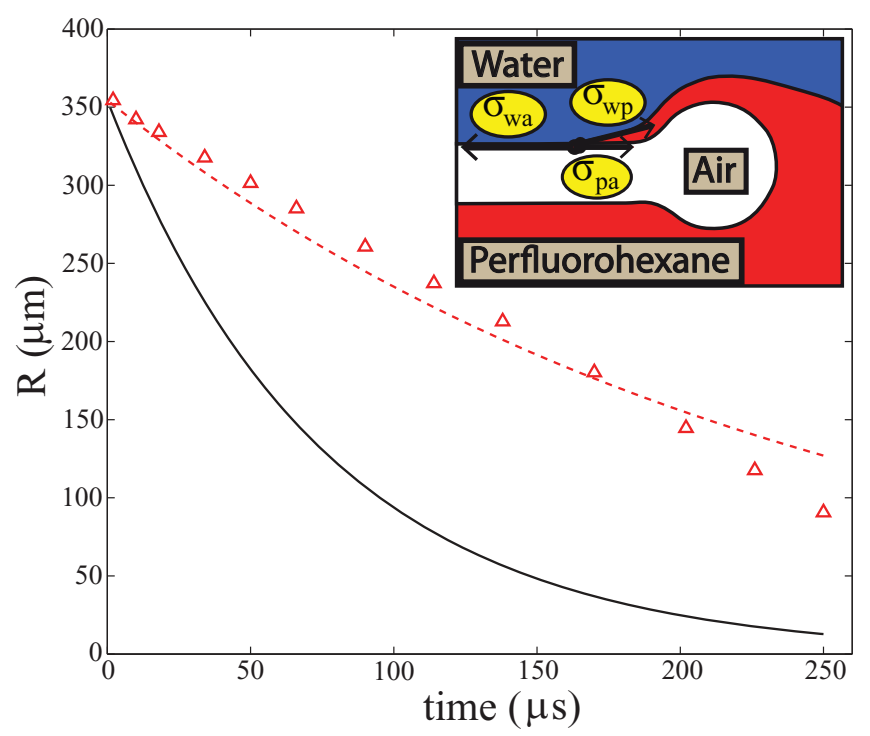

FIG. 4. (Color online) Radius of the central disk as a function of time, for a water drop impacting on a perfluorohexane pool (with $h=250 \mu \mathrm{m}, H=42.5 \mathrm{~cm}$, and $D_{v} / D_{h}=0.80$ ). The dashed line indicates Eq. (1) with perfluorohexane liquid properties and the solid line is with water properties, both with $C=0.94$.

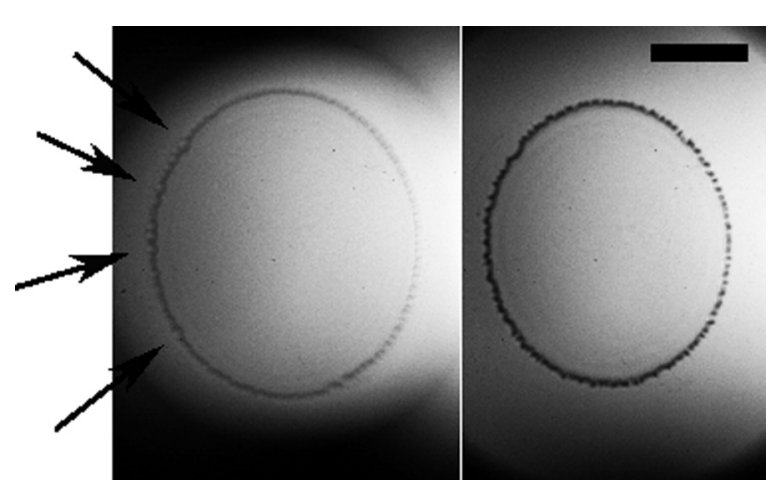

FIG. 5. Early contact of a water drop impacting a water film at $U=2.8 \mathrm{~m} / \mathrm{s}$. Taken from a $250-\mathrm{kfps}$ video clip $(h=500 \mu \mathrm{m})$, with a $2-\mu \mathrm{s}$ exposure. Frames are spaced by $4 \mu \mathrm{s}$. The arrows point at prominent circles where the liquids first make contact. The scale bar is $200 \mu \mathrm{m}$.

the dynamics should change when the relative strength of these three surface tensions are altered and more experiments, with various liquid combinations, are needed to fully understand this configuration.

Edge undulations. Another interesting observation is of the onset of the edge undulations that develop for the waterwater case. These undulations were studied in side view by Thoroddsen et al. [3], but in the bottom view we can observe the very earliest contact, within $1-\mu$ s time resolution. The earliest image in Fig. 2(a) shows isolated circles (arrow), which may be contacts driven by van der Waals forces as the film between the two liquid surfaces reaches below $100 \mathrm{~nm}$ in thickness $[15,35,36]$. We can observe microbubbles left behind near this initial contact, but their sizes are too small for characterization in the current spatial resolution [37,38]. Figure 5 shows another realization where these undulations are visible all around the first contact circle. In this case there are 85 distinct structures in the second frame, corresponding to a wavelength of $\simeq 20 \mu \mathrm{m}$ along the periphery. It is known that the first contact of a drop with a smooth solid surface leaves behind a dark ring of microbubbles (Figs. $2 \mathrm{a}$ and $3 \mathrm{~g}$ in Ref. [4]). Imaging using total internal reflection [23] and interferometry [22,27] has directly observed such isolated ruptures for low-impact-velocity drops onto a solid surface. Our images show similar local contacts for liquid-liquid impacts, at much higher impact velocity. However, these undulations are not observed in the water on ethanol impacts, as well as in the immiscible impacts onto perfluorohexane [Figs. 2(c) and 2(d)]. This could be due to the stabilizing effect of the sudden tangential stresses at the free surface or the lower surface tension of the pool, but imaging at even higher frame rates will be needed to resolve the origin and absence of these undulations.

Summary. Herein we demonstrated that the contraction of an air disk is governed by the weaker of the two surface tensions. For the miscible case, this dominance relies conceptually on Marangoni stresses along the free surface pulling a thin layer of ethanol over the water surface, the overall dynamics therefore only seeing the ethanol surface tension. These results are consistent with that observed in the inverse configuration of a water and an ethanol drop coalescing [28]. We suggest similar 
dynamics for the immiscible case. We furthermore observed azimuthal undulations for a water drop impacting a pool of water, which are stabilized for a pool of lower surface tension.
Acknowledgments. We thank T. G. Etoh and K. Takehara at Kinki University in Osaka, Japan, for the use of their highspeed video cameras for many of the experiments.
[1] S. Chandra and C. T. Avedisian, Proc. R. Soc. London Ser. A 432, 13 (1991).

[2] B. Peck and L. Sigurdson, Phys. Fluids 6, 564 (1994).

[3] S. T. Thoroddsen, T. G. Etoh, and K. Takehara, J. Fluid Mech. 478, 125 (2003).

[4] S. T. Thoroddsen, T. G. Etoh, K. Takehara, N. Ootsuka, and Y. Hatsuki, J. Fluid Mech. 545, 203 (2005).

[5] S. Mandre, M. Mani, and M. P. Brenner, Phys. Rev. Lett. 102, 134502 (2009).

[6] M. Mani, S. Mandre, and M. P. Brenner, J. Fluid. Mech. 647, 163 (2010).

[7] P. D. Hicks and R. Purvis, J. Fluid Mech. 735, 120 (2013).

[8] M.-J. Thoraval, K. Takehara, T. G. Etoh, and S. T. Thoroddsen, J. Fluid Mech. 724, 234 (2013).

[9] P. D. Hicks and R. Purvis, Phys. Fluids 23, 062104 (2011).

[10] H. N. Oguz and A. Prosperetti, J. Fluid Mech. 203, 149 (1989).

[11] F. E. C. Culick, J. Appl. Phys. 31, 1128 (1960).

[12] M. P. Brenner and D. Gueyffier, Phys. Fluids 11, 737 (1999).

[13] L. Gordillo, G. Agbaglah, L. Duchemin, and C. Josserand, Phys. Fluids 23, 122101 (2011).

[14] H. Lhuissier and E. Villermaux, Phys. Fluids 23, 091705 (2011).

[15] É. Reyssat and D. Quéré, Europhys. Lett. 76, 236 (2006).

[16] N. Savva and J. W. M. Bush, J. Fluid Mech. 626, 211 (2009).

[17] J. Zou, C. Ji, B. G. Yuan, X. D. Ruan, and X. Fu, Phys. Rev. E 87, 061002(R) (2013).

[18] S. T. Thoroddsen and J. Sakakibara, Phys. Fluids 10, 1359 (1998).

[19] J. de Ruiter, J. M. Oh, D. van den Ende, and F. Mugele, Phys. Rev. Lett. 108, 074505 (2012).

[20] P. D. Hicks, E. V. Ermanyuk, N. V. Gavrilov, and R. Purvis, J. Fluid Mech. 695, 310 (2012).

[21] J. S. Lee, B. M. Weon, J. H. Je, and K. Fezzaa, Phys. Rev. Lett. 109, 204501 (2012).
[22] M. M. Driscoll and S. R. Nagel, Phys. Rev. Lett. 107, 154502 (2011).

[23] J. M. Kolinski, S. M. Rubinstein, S. Mandre, M. P. Brenner, D. A. Weitz, and L. Mahadevan, Phys. Rev. Lett. 108, 074503 (2012).

[24] R. C. A. van der Veen, T. Tran, D. Lohse, and C. Sun, Phys. Rev. E 85, 026315 (2012).

[25] J. O. Marston, I. U. Vakarelski, and S. T. Thoroddsen, J. Fluid Mech. 680, 660 (2011).

[26] W. Bouwhuis, R. C. A. van der Veen, T. Tran, D. L. Keij, K. G. Winkels, I. R. Peters, D. van der Meer, C. Sun, J. H. Snoeijer, and D. Lohse, Phys. Rev. Lett. 109, 264501 (2012).

[27] Y. Liu, P. Tan, and L. Xu, J. Fluid Mech. 716, R9 (2013).

[28] S. T. Thoroddsen, B. Qian, T. G. Etoh, and K. Takehara, Phys. Fluids 19, 072110 (2007).

[29] A. D. Dussaud and S. M. Troian, Phys. Fluids 10, 23 (1998).

[30] M. Santiago-Rosanne, M. Vignes-Adler, and M. G. Velarde, J. Colloid Interface Sci. 191, 65 (1997).

[31] T. G. Etoh et al., IEEE Trans. Electron Devices 50, 144 (2003).

[32] M.-J. Thoraval et al., Phys. Rev. Lett. 108, 264506 (2012).

[33] A. A. Castrejón-Pita, J. R. Castrejón-Pita, and I. M. Hutchings, Phys. Rev. E 86, 045301(R) (2012).

[34] See Supplemental Material at http://link.aps.org/supplemental/ 10.1103/PhysRevE.88.061001 for video clips.

[35] S. T. Thoroddsen, M.-J. Thoraval, K. Takehara, and T. G. Etoh, J. Fluid Mech. 708, 469 (2012).

[36] T. Tran, H. De Maleprade, C. Sun, and D. Lohse, J. Fluid Mech. 726, R3 (2013).

[37] S. T. Thoroddsen, K. Takehara, and T. G. Etoh, Phys. Fluids 22, 051701 (2010).

[38] D. L. Keij, K. G. Winkels, H. Castelijns, M. Riepen, and J. H. Snoeijer, Phys. Fluids 25, 082005 (2013). 